\title{
MODE JUMPING OF AN ISOGRID PANEL UNDER QUASI-STATIC COMPRESSION
}

\author{
Danniella M. Muheim \\ Member AIAA \\ Aerospace Engineer, Analytical and Computational Mechanics Branch \\ NASA Langley Research Center, Hampton, VA, 23681 \\ Eric R. Johnson \\ Member AIAA and ASME \\ Professor, Aerospace and Ocean Engineering \\ Virginia Polytechnic Institute and State University, Blacksburg, VA, 24061-0203
}

\begin{abstract}
A wide column test of a composite isogrid panel subjected to quasi-static, axial compression is modeled with a hybrid-static dynamic computational method. The data from the test panel exhibited discontinuous responses in the compressive load for slowly increased end-shortening. The computational model was developed to corroborate these discontinuities with the phenomenon of mode jumping. Mode jumping refers to the transient response of the panel from an unstable bifurcation point on a postbuckled equilibrium path to a second stable equilibrium state on a new equilibrium path. On the new equilibrium path, both the analysis and test show that the panel can resist increased endshortening beyond that of the unstable critical point. Fair agreement is achieved between the analysis and test.
\end{abstract}

\section{Introduction}

The postbuckling response of geodetic and isogrid stiffened structures under quasi-static compression loading has received less attention than the buckling of these structures. This lack of attention may be due to the common approach to design so that a geodetic structure fails in a global buckling mode rather than in a local buckling mode. However, Refs. 1-3, indicated that when local buckling occurred first it was not catastrophic, and it was possible to increase the applied load until total collapse occurred. Koury et al. ${ }^{1}$, noted that one of their panels underwent three different buckle patterns under increasing load prior to the catastrophic failure of a single stiffener. Heard et al. ${ }^{2}$, noted that a materially nonlinear analysis of the postbuckled response of an aluminum isogrid cylinder exhibited repeated changes

This material is declared a work of the U.S. Government and is not subject to copyright protection in the United States. in buckle pattern during monotonic loading. The cylinder buckle patterns were of progressively shorter wavelength, and the changes in each pattern were accompanied by brief drops in strain. The test results ${ }^{2}$, however, did not exhibit these transient changes in buckle pattern.

Transient changes in the postbuckled deformation states under monotonically increasing quasi-static, endshortening were first observed in a compression test of a multi-bay, flat aluminium plate by Stein ${ }^{4}$. This phenomenon is now called mode jumping. Mode jumping refers to the transient response of a structure from an unstable bifurcation point on a postbuckled equilibrium path to a second stable equilibrium state on a new equilibrium path. The structure can carry increasing static compressive loads beyond that of the unstable critical point on the new equilibrium path. Thus, the structure exhibits postbuckling strength along the new stable equilibrium path. Since it is difficult to use static path following methods to locate disconnected equilibrium paths, combined static and dynamic analyses can model best conditions observed in tests. A hybrid static-dynamic computational approach was developed and used by Riks, Rankin and Brogan ${ }^{5}$, and later used by others $^{6,7}$ to model one bay of Stein's aluminum plate. Others have used a hybrid static-dynamic approach to model the response of composite cylinders ${ }^{8,9}$, composite cylinders with cutouts ${ }^{10}$, and cracked aluminum cylinders ${ }^{11}$.

To the authors' knowledge, there is no reported work in the literature on mode jumping of isogrid panels. The objective of this paper is to use a hybrid staticdynamic computational approach to corroborate that the discontinuities observed in the load-shortening response of an isogrid test panel are associated with the mode jumping phenomenon. The procedures to transition between static and dynamic analyses and to select the 
damping coefficients differ somewhat with those in Refs. 5-11. The following sections are included in the paper: wide column test of the composite isogrid panel, hybrid static-dynamic method, finite element model, results and discussion, and some concluding remarks.

\section{Wide column compression tests}

A series of composite isogrid panels of rectangular planform were tested under quasi-static, uniaxial compression as wide columns. That is, the two opposite lateral edges of the panels were free, and the loaded edges were secured in an iron frame fixture. The panels were laminated from IM7/977-2 graphite-epoxy with a skin lay-up of $\left[ \pm 60 / 0_{2}\right]_{2 s}$, and a stiffener lay-up of $[0]_{8}$. These isogrid panels were manufactured by the United States Air Force Phillips Laboratory for composite launch vehicle components ${ }^{1}$. One of the delivered panels, labeled P23, was cut to form additional test articles denoted P23RA, P23RB, P23LA, and P23LB as shown in Fig. 1(a). Test articles LA and LB had three axial stiffeners, and RB and RA had one axial stiffener. Notice in Figs. 1(a)-(b) that distinct polygonal skin regions defined by the stiffener pattern were not symmetric about the axial stiffeners. This asymmetry was due to the manufacturing technique used to avoid stiffener material build-up by 'off-setting' the three intersecting stiffeners.

The longitudinal ends of the test articles were secured in iron fixtures filled with an aluminum epoxy potting compound and were ground flat and parallel. The instrumented specimens were placed between the platens of a hydraulic testing machine with an axial load capacity of 120 kips $(533.8 \mathrm{kN})$. The testing machine was operated in displacement control with the rate of end-shortening specified as $0.02 \mathrm{in} . / \mathrm{min}$. $(0.508 \mathrm{~mm} /$ $\min$.).

Each of the test panels exhibited discontinuities or "jumps" in the load versus end-shortening plots, and other response plots. Only panel P23RA is selected for the analyses presented here. This small panel had one continuous axial stiffener in the center, four off-axis stiffeners that terminated at the free edges of the panel, and had its three-stiffener intersections contained in the potting material of the end-fixtures. Panel P23RA had planform dimensions of approximately $3.75 \mathrm{in}$. by $6 \mathrm{in}$. ( $95.25 \mathrm{~mm}$ by $152.4 \mathrm{~mm}$ ) The nominal cross-sectional dimensions of the stiffeners are $0.64 \mathrm{in.}(16.26 \mathrm{~mm})$ in height and $0.0455 \mathrm{in} .(1.156 \mathrm{~mm})$ in thickness. The nominal skin thickness is 0.0564 in. (1.433 mm).
Fourteen electrical resistance strain gages were bonded to the skin and axial stiffener. These gages are numbered one to fourteen as shown in Fig. 1(b), and are identified as SG1 to SG14 in the text. Gages SG1 and SG3 measured back-to-back axial strains in the left skin cell, and SG5 and SG7 measured back-to-back axial strains in the right skin cell. In Fig. 1(b), back side gage numbers are shown in parentheses. Gages SG13 and SG14 measured back-to-back strains at the center of the web of the axial stiffener in a direction normal to the skin. One direct current differential transducer (DCDT) was used to monitor the axial displacement of the panel at the movable platen. The second and third DCDTs monitored the out-of-plane displacements at the center of the left skin cell and at the center of the right skin cell, and these displacements are denoted by $w_{L}$ and $w_{R}$, respectively. Measurement of $w_{L}$ is coincident with gage SG1, and measurement of $w_{R}$ is coincident with gage SG5.

\section{Hybrid static-dynamic method}

The hybrid static-dynamic approach for the nonlinear response of the panel consists of three steps. First, a static analysis is performed to establish the equilibrium path on the load end-shortening response plot that emanates from the origin. Stability analyses of these states on this equilibrium path are conducted to locate the unstable critical point. Second, a dynamic analysis is initiated at this unstable critical point, which includes dissipative forces, to represent the transient mode jump of the panel to the vicinity of a new stable equilibrium state, assuming the new asymptotically stable state exists. Third, a second sequence of static analyses is undertaken along this new stable equilibrium path.

To model the response of the panel, we assume the material law is linear elastic, the strain-displacement relations referred to the reference state are nonlinear, the external dissipative forces are due to linear viscous damping, and that any external loads are conservative and independent of the displacements; i.e., deadweight external loads. Using the finite element method, the continuum representation of the panel is discretized. Let $\boldsymbol{U}$ denote the generalized nodal displacement vector, $\boldsymbol{U}$ the velocity vector, and let $\ddot{U}$ denote the acceleration vector. The $\operatorname{dot}\left({ }^{\circ}\right)$ denotes differentiation with respect to time. The equations of motion are

$$
M U+D \dot{U}+f^{i n t}(U)=\lambda f^{e x t}
$$

where $\boldsymbol{M}$ is the symmetric and positive definite mass matrix, $\boldsymbol{D}$ is the symmetric and positive definite damp- 
ing matrix, $f^{i n t}(U)$ is the internal force vector, $\lambda$ is the load factor, and $\boldsymbol{f}^{\text {ext }}$ is the external load vector. Controlled, proportional external loading is assumed in Eq. (1). Since the material is elastic, the components of the internal force vector are the partial derivatives of the strain energy with respect to the corresponding nodal displacement.

Setting the time derivatives of $\boldsymbol{U}$ to zero in Eq. (1), yields the governing nonlinear static equilibrium equations. These equilibrium equations are solved iteratively by Newton's method, which leads to the following sequence of linear equations for the incremental displacement vectors $\Delta \boldsymbol{U}^{(k)}$, where $k$ is the current iteration, and $k=1,2, \ldots$,

$$
\begin{gathered}
\boldsymbol{K}_{\boldsymbol{T A N}} \Delta \boldsymbol{U}^{(k)}=\boldsymbol{R}^{(k)}=\lambda \boldsymbol{f}^{\text {ext }}-\boldsymbol{f}^{\boldsymbol{i n t}}\left(\boldsymbol{U}^{(k-1)}\right) \\
\boldsymbol{U}^{(k)}=\boldsymbol{U}^{(k-1)}+\Delta \boldsymbol{U}^{(k)}
\end{gathered}
$$

In Eq. (2) the tangent stiffness matrix is given by

$$
\boldsymbol{K}_{\boldsymbol{T A N}}\left[\boldsymbol{U}^{(k-1)} ; \lambda\right]=\partial \boldsymbol{f}^{\text {int }} /\left.\partial \boldsymbol{U}\right|_{\boldsymbol{U}^{(k-1)}}
$$

and $\boldsymbol{R}^{(k)}$ is the residual force vector. The initial guess $\boldsymbol{U}^{(0)}$ is the equilibrium displacement vector from the previous load state. If the iterations converge, then the residual force vector is very close to the null vector with respect to a specified error tolerance. When the sequence $\left\{\boldsymbol{U}^{(1)}, \boldsymbol{U}^{(2)}, \ldots\right\}$ converges, the limit $\boldsymbol{U}^{*}(\lambda)$ is the equilibrium displacement vector for the specified load factor $\lambda$.

As the system as represented by Eq. (1) is purely and completely dissipative ${ }^{12}$, the energy method can be used to analyze the stability of an equilibrium state. Thus, the stability of an equilibrium state is determined by the nature of the quadratic form given by the second variation of the total potential energy. Let $\delta^{2} \Pi$ denote the second variation of the total potential energy, which is equal to the second variation of the strain energy for deadweight loading. Since the first partial derivatives of the strain energy with respect to the displacements gives the components of the internal force vector, then the tangent stiffness matrix in Eq. (4) is equivalent to computing the second partial derivatives of the strain energy with respect to the displacements. Consequently, the second variation of the total potential energy can be written as

$$
\delta^{2} \Pi=\left(\delta \boldsymbol{U}^{T} \boldsymbol{K}_{\text {TAN }} \delta \boldsymbol{U}\right) / 2
$$

where $\boldsymbol{K}_{\boldsymbol{T A N}} \equiv \boldsymbol{K}_{\boldsymbol{T A N}}\left(\boldsymbol{U}^{*} ; \lambda\right), \delta \boldsymbol{U}$ is any kinematically admissible variation of the displacement vector about the equilibrium state, and the superscript $T$ denotes matrix transpose. Eigenvalues of the tangent stiffness matrix at an equilibrium state determined its stability. For the equilibrium path on the load versus endshortening response plot that emanates from the origin, we seek the first unstable critical state encountered when monotonically increasing the load factor, $\lambda$, from zero. For a perfect system, there may be stable critical states corresponding to bifurcation points as $\lambda$ is increased. In the numerical analysis, we determined the first unstable critical point if no stable equilibrium state were found in the vicinity of a critical point on the loadshortening plot. Let $S_{c r}$ denote this first unstable critical state at the corresponding load factor $\lambda_{c r}$

A nonlinear dynamic analysis is initiated from the state $S_{c r}$ with the load factor fixed at $\lambda_{c r}$ by specifying a small initial velocity times the eigenvector associated with the zero eigenvalue of the tangent stiffness matrix. The time derivatives of the internal forces, displacements, and velocities are approximated in the time domain with an implicit time integration scheme. Proportional damping ${ }^{13}$ is assumed; i.e.,

$$
\boldsymbol{D}=\alpha \boldsymbol{M}+\beta \boldsymbol{K}_{\text {TAN }}(\boldsymbol{U} ; \lambda),
$$

where $\alpha$ and $\beta$ are the mass and stiffness scalar coefficients of proportionality. Matrix $\boldsymbol{D}$ is updated for each iteration at a particular time step of the dynamic analysis with a full Newton-Raphson scheme. Coefficients $\alpha$ and $\beta$ are selected by analogy to a linear, single degree-offreedom oscillator. For this linear oscillator, the scalar product $M^{-1} D$ is equal to $2 \zeta \omega$ and the scalar product $M^{-1} K$ equals $\omega^{2}$, where $\zeta$ is the dimensionless damping factor and $\omega$ is the undamped natural frequency in radians per second. Thus, for proportional damping of the linear oscillator, Eq. (6) reduces to

$$
2 \zeta \omega=\alpha+\beta \omega^{2} .
$$

We define mass and stiffness damping factors $\zeta_{M}$ and $\zeta_{K}$, respectively by $\alpha=\zeta_{M} \omega$ and $\beta=\zeta_{K} / \omega$ such that Eq. (7) leads to $2 \zeta=\zeta_{M}+\zeta_{K}$. The underdamped case is used to specify $\zeta_{M}$ and $\zeta_{K}$ such that $0<\zeta<1$. Let $f$ be an undamped natural frequency in Hertz, then the coefficients $\alpha$ and $\beta$ are given by

$$
\alpha=\zeta_{M} 2 \pi f \quad \beta=\zeta_{K} /(2 \pi f)
$$

The frequency used in the analysis is the lowest nonzero vibration frequency at the critical state $S_{c r}$ By definition, the fundamental frequency at state $S_{c r}$ vanishes and its eigenmode coincides with the buckling mode, so that the lowest nonzero frequency is the second frequency. 
After initiating the motion at state $S_{c r}$ the trajectory of the motion in phase space approaches an asymptotically stable equilibrium state denoted as $S_{1}$, assuming this state exists. The trajectory is considered to have arrived in the vicinity of state $S_{1}$ once the kinetic energy remains less than $1 \%$ of the peak kinetic energy in the transient response. Let $T_{A}$ denote the time of arrival of the trajectory in the vicinity of state $S_{1}$. The displacement vector in the transient analysis at $T_{A}$ is used as the initial guess $\boldsymbol{U}^{(0)}$ in the Newton's iteration to determine the displacement vector for equilibrium state $S_{1}$. A geometrically nonlinear static analysis along the secondary path is continued until additional instabilities are encountered, or the analysis is otherwise completed.

\section{Finite element model}

All finite element analyses were performed using the Structural Analysis of General Shells (STAGS) finite element software ${ }^{13}$, and the 410 element $^{14}$. The 410 element is a flat, 4-node quadrilateral element with three translations $(u, v, w)$, and three rotations $\left(\beta_{x}, \beta_{y}, \beta_{z}\right)$ at each node. This element is formulated from the Kirchhoff-Love hypotheses for small strains, and it is implemented using a co-rotational procedure to account for large rotations and displacements.

The finite element model of panel P23RA referred to Cartesian coordinates $x, y$, and $z$ is shown in Fig. 2. The directions of positive displacements and rotations are also indicated in this figure. Along the top edge of the model, a uniform axial displacement in the negative $x$-direction, denoted by $\bar{u}$, was specified at all nodes by a series of multiple-point constraint equations. All other degrees-of-freedom (DOF) along the top edge were specified to vanish. The bottom edge of the model was fixed. The panel within the end fixtures was modeled by specifying all DOFs to vanish except for displacement $u$. A buckling analysis from a linear prebuckling equilibrium configuration of the perfect panel was used to judge if the finite element mesh had sufficient fidelity. Mesh refinement was terminated once an additional refinement indicated no change in the first ten buckling loads and modes. There were 2,676 nodes and 2,605 elements, and this mesh is depicted in Fig. 2.

The material properties used in the analysis are listed in Table 1. The nominal value of the longitudinal modulus $E_{1}$ supplied by manufacturer was changed, by less than $5 \%$, such that the stiffness from a linear analysis with the refined mesh matched the slope of the test data on the load-shortening plot near the origin.

All geometrically nonlinear analyses, static or dynamic, were performed under controlled shortening. Park's form of implicit, linear multi-step method ${ }^{15}$ was used with a constant time step, denoted $D T$, for all dynamic analyses. The default STAGS convergence tolerance on the displacement norm and the residual norm ${ }^{13}$ was reduced to $D E L E X=1 \times 10^{-7}$, while the eigenvalue tolerance ${ }^{13}$ was reduced to $D E L E V=1 \times 10^{-5}$.

\section{Results and Discussion}

\section{Buckling analysis of the linear equilibrium state}

The critical value of the end-shortening for the linear prebuckling equilibrium configuration of the perfect model of panel P23RA was $\bar{u}_{\text {cr }}=0.005266$ in. (0.1338 $\mathrm{mm})$ as predicted by analysis. The associated critical load was 4,448 lbs. $(19.78 \mathrm{kN})$. Let $\Delta$ denote the normalized end-shortening defined by $\Delta=\bar{u} / \bar{u}_{\mathrm{cr}}$ such that $\Delta=1$ corresponds to the critical end-shortening predicted for the perfect structure from a linear prebuckling equilibrium configuration. Also, we normalized the load factor by the relation $\lambda \rightarrow \lambda / \lambda_{\text {cr }}$ such that $\lambda=1$ corresponds to the critical load. Test data was similarly normalized.

\section{$\underline{\text { Test results }}$}

The load-shortening response of the panel P23RA from the test is shown on the plot of load factor versus the normalized end-shortening in Fig. 3(a). As shown in Fig. 3(a), one discontinuity in the response occurs at $(\Delta$, $\lambda)=(1.530,1.400)$, and a second one occurs at $(\Delta, \lambda)=$ $(2.612,1.983)$. A magnification of the plot in Fig. 3(a) near $(\Delta, \lambda)=(1.530,1.400)$ is shown in Fig. 3(b). At the initiation of the first jump, the load is $6,227 \mathrm{lbs}$. (27.7 $\mathrm{kN}$ ), and at the end of the jump, the load factor reduces to $\lambda=1.393$, which corresponds to a decrease in load of $28 \mathrm{lbs}$. (125 N). At the initiation of the second jump, the load is 8,818 , lbs. $(39.2 \mathrm{kN})$, and at the end of the jump, the load factor reduces to $\lambda=1.910$, which corresponds to a decrease in load of $323 \mathrm{lbs}$. $(1.44 \mathrm{kN})$. The load factor versus out-of-plane displacement at the centers of left skin cell and the right skin cell are shown in Fig. 4(a). A magnification of the plot in Fig. 4(a) near $\lambda=$ 1.400 is shown in Fig. 4(b). Note that the discontinuity in the deflection response at $\lambda=1.400$ only occurs in the left skin cell of the panel and not on the right skin cell. The back-to-back axial strain gage data for the left skin cell in Fig. 5(a) and the right skin cell in Fig. 5(b), also 
corroborates that the deflection discontinuity in the response occurs only in the left skin cell. Back-to-back gages SG13 and SG14 on the web of the axial stiffener indicate a jump and change of sign of the strain at $\lambda=$ 1.400 as shown in Fig. 6 . Hence, at $\Delta=1.530$ both the skin on the left side of the axial stiffener and the stiffener itself exhibit jumps in their displacement response. However, the skin on the right side of the stiffener does not change its deformation pattern.

\section{Analysis for the initial unstable critical state}

To model the loss of stability near $\Delta=1.530$ observed in the test, we had to include a geometric imperfection in the finite element analyses. A geometrically nonlinear analysis of the perfect panel indicated an unstable critical point near $\Delta=1$ and not at $\Delta=1.530$. The eigenvectors from the linear prebuckling equilibrium configuration at $\Delta=1$ were used to form the initial imperfection as no measured surface imperfection data was available from the test. An initial geometric imperfection ${ }^{13,16}$ is represented by a stress-free displacement state $\boldsymbol{U}_{0}$, specified by

$$
\boldsymbol{U}_{0}=a_{1} \boldsymbol{U}_{1}+a_{2} \boldsymbol{U}_{2}+a_{3} \boldsymbol{U}_{3}+a_{4} \boldsymbol{U}_{4}
$$

where $\boldsymbol{U}_{i}, i=1,2,3,4$, are the first four buckling modes. Each mode is normalized in STAGS such that its maximum displacement component is unity. The participation factors $a_{i}$ were determined by trial, with the objective of finding an unstable state on the equilibrium path of the imperfect model near $\Delta=1.530$. Employing a geometrically nonlinear static analysis, we found that when

$$
a_{1}=a_{2}=a_{3}=-a_{4} \quad\left|a_{i}\right|=0.1 \mathrm{~h}
$$

where $h$ is the skin thickness, that one negative eigenvalue in $\boldsymbol{K}_{\text {TAN }}$ occurred above a value of $\Delta=1.762$, which corresponds to $\lambda=1.576$. At the next shortening increment, a negative root was found, therefore the critical state was bounded, and $(\Delta, \lambda)=(1.762,1.576)$ is a stable equilibrium state $S_{s}$ near the critical state $S_{c r}$ The initial geometry of the imperfect panel corresponding to Eqs. (9) and (10) is shown in Fig. 7. The deformed panel at equilibrium state $S_{s}$ is shown in Fig. 8. An eigenvalue analysis of the tangent stiffness matrix $\boldsymbol{K}_{T A N}$ at stable state $S_{S}$ is performed to estimate the shortening, and corresponding load factor at the unstable critical state $S_{c r}$. The first two eigenvalues of $\Delta$ at state $S_{S}$, and the projected values of $\Delta$ to estimate subsequent critical states are listed in Table 2. Thus, from the lowest projection in Table 2 we estimate that the critical normalized shortening at state $S_{c r}$ is $\Delta_{c r}=1.762\left(1+e_{1}\right)$
$=1.764$, where $e_{1}$ is the lowest eigenvalue of $\boldsymbol{K}_{\text {TAN }}$ at state $S_{s}$, and the corresponding load factor is $\lambda_{c r}=$ 1.578. The equilibrium state corresponding to $(\Delta, \lambda)=$ $(1.762,1.576)$ on the equilibrium path, which is denoted $S_{s}$, is stable, but it is near the initial unstable state $S_{c r}$ at $\left(\Delta_{c r}, \lambda_{c r}\right)=(1.764,1.578)$.

Transient response initiated at the unstable critical state

A mode jump is initiated for the imperfect panel model under controlled shortening. The equilibrium displacements are those from state $S_{S}$, but the normalized end-shortening was specified as $\Delta=1.770$, which is $0.4 \%$ greater than the estimated critical shortening value. The distribution of the initial velocity is specified as the eigenmode predicted for equilibrium state $S_{c r}$ which was scaled so that the component with the largest magnitude was $0.01 \mathrm{in} . / \mathrm{s}(0.254 \mathrm{~mm} / \mathrm{s})$. This eigenmode is the same shape as the first vibration mode, which is shown in Fig. 9(a). The resultant initial velocity magnitude was $0.035 \mathrm{in} . / \mathrm{s}(0.89 \mathrm{~mm} / \mathrm{s})$.

To estimate the proportional damping factors $\alpha$ and $\beta$ in Eq. (8), a linear vibration analysis was performed at the equilibrium state $S_{s}$ to determine the natural frequencies. The first two natural frequencies are listed in Table 2, and the corresponding vibration modes are shown in Figs. 9(a) and 9(b), respectively. The frequency $f_{2}$ is representative of the first non-zero frequency of the theoretically unstable critical state and was used in Eq. (8). We specified the mass and stiffness damping factors in Eq. (8) as $\zeta_{M}=0.06$ and $\zeta_{K}=0.171$, and calculated $\alpha=493 / \mathrm{s}$ and $\beta=21 \times 10^{-6}$ s. The effective level of damping was $\zeta=0.116$, which is within the recommended range of $0.05<\zeta<0.20$ from Ref. 5. We note that researchers ${ }^{5-11}$ have used the frequencies of either the loaded or unloaded configuration to specify $\alpha$ and $\beta$.

A constant time step of $D T=20 \times 10^{-6} \mathrm{~s}$, was used in the Park's numerical integration method. A maximum value of kinetic energy of $2.45 \times 10^{-3}$ in.-lb. $\left(277 . \times 10^{-6} \mathrm{~J}\right)$ occurred at time $T=2.04 \times 10^{-3} \mathrm{~s}$. Using the $1 \%$ criterion mentioned earlier, the time of arrival in the vicinity of state $S_{1}$ was $T_{A}=8.08 \times 10^{-3} \mathrm{~s}$. The strain energy decreased from an initial value of 35.126 in.-lb. (3.969 $\mathrm{J})$ to a value of $35.116 \mathrm{in.}-1 \mathrm{~b}$. (3.967 J) at $T_{A}$.

The change in load factor to $\lambda=1.734$ shown in Fig. 3(b) occurred during the transient analysis because the end-shortening was fixed in value. The load factor of 1.578 at the initial unstable critical state $S_{c r}$ decreased to 
1.570 at state $S_{1}$, which is a $35 \mathrm{lb} .(155 \mathrm{~N})$ decrease in load. As shown in Fig. 4(b), the skin cell deflections $w_{L}$ and $w_{R}$ both increase in value through the mode jump. Compare the deformed shape of the axial stiffener before the mode jump in Fig. 8 to its shape after the mode jump in Fig. 10. This comparison reveals that there is a reversal in the curvature of the web through its height at the center of the stiffener, as a result of a shift in the deformation pattern along the length of the stiffener.

\section{$\underline{\text { Static analysis on the new equilibrium path }}$}

A geometrically nonlinear static analysis of the imperfect isogrid panel was re-initiated at a normalized end-shortening of $\Delta=1.7702$ using the displacement vector from the transient state at $T=T_{A}$ as the initial guess for a new equilibrium state along the secondary postbuckling path. This is an increase in $\Delta$ of only $0.01 \%$ with respect to the value of 1.7700 specified during the transient analysis. A total of four iterations were required to converge at $\Delta=1.7702$ at this first step of the nonlinear static analysis. The load was incremented until the onset of a new instability was detected at $(\Delta, \lambda)=(6.971,3.355)$ along this new postbuckling path.

\section{Comparison of test and analysis}

The wide column test of the composite isogrid panel under slowly increased end-shortening, and the hybrid static-dynamic analysis of this panel, both exhibit an abrupt change in shape of the panel where the load jump occurs, followed by continued loading after the load jump. The analysis predicted the critical state $S_{c r}$ at ( $\Delta$, $\lambda)=(1.762,1.578)$, while the corresponding values from the test are $(1.530,1.40)$. That is, the predicted normalized shortening is $15 \%$ greater, and the load factor is $13 \%$ greater, than the corresponding values from the test. The decrease in load through the mode jump predicted by the analysis was $35 \mathrm{lb}$. (156 N), or $25 \%$ more than the drop recorded in the test of $28 \mathrm{lbs}$. $(125 \mathrm{~N})$. The slope of the load-shortening response following the mode jump predicted by the analysis is less than that from the test as shown in Fig. 3(b). An unstable state was predicted on the new equilibrium path from the analysis at $(\Delta, \lambda)=(6.971,3.355)$, but the second mode jump in the test occurred at $(\Delta, \lambda)=$ $(2.612,1.983)$.

The out-of-plane displacements of the left $\left(w_{L}\right)$ and right $\left(w_{R}\right)$ skin cells predicted from the analysis and those measured in the test are in reasonable agreement as is shown in Figs. 4(a) and 4(b). However, the test data indicated that the displacement $w_{L}$ decreased slightly through the mode jump and displacement $w_{R}$ did not change, while the analysis predicted both the displacements $w_{L}$ and $w_{R}$ increased through the mode jump as shown in Fig. 4b. At the load jump, the abrupt change in the strains from gages SG13 and SG14 shown in Fig. 6 means there is change in curvature of the stiffener through its height at mid-span, which is also demonstrated by the deformation change predicted from the analysis as shown in Fig. 8 and Fig. 10.

\section{Concluding remarks}

The correlation of the hybrid static-dynamic nonlinear finite element analyses to the wide column, composite isogrid test article measurements indicates and corroborates that the discontinuities observed in the response under monotonically increasing quasi-static shortening are associated with the phenomenon of mode jumping. To achieve the correlation with the test, the mode jumping analysis required: (1) locating the unstable bifurcation point on the equilibrium path emanating from the origin on the load end-shortening response plot of the imperfect panel; (2) a transient dynamic analysis initiated at the unstable bifurcation point which included viscous proportional damping and an initial velocity in the shape of the buckling mode; and (3) re-establishment of equilibrium on the new equilibrium path using as an initial estimate of the displacement the displacement obtained from the transient analysis when the kinetic energy remained less than $1 \%$ of its peak value.

The test results showed that the discontinuity in the response was manifested by a jump in the lateral deflection of the left side skin cell and a jump in the bending response of the central axial stiffener. The right skin cell did not exhibit a jump in response. However, the analysis predicted that both the left and right side skin cell deflections increased through the mode jump. The drop in the compressive force at a fixed endshortening in the mode jump predicted from the analysis was $25 \%$ more than the recorded experimental results, which is a good correlation. However, the analysis over predicted the force and end-shortening at the critical equilibrium state, from which the mode jump was initiated, by approximately $15 \%$.

Subsequent loading along the new static equilibrium path in the analysis indicated an instability at a normalized shortening of 6.971, which exceeds the normalized shortening of 3.355 for the secondary instability recorded in the test. The discrepancy between 
the analysis and test for the second mode jump is likely due to material damage that may have occurred in the test panel that is not modeled in the analysis.

\section{Acknowledgements}

The authors acknowledge technical discussions with Dr. N. F. Knight, Jr. of Veridian Systems Division, Yorktown, VA, and Dr. C.C. Rankin of Lockheed Martin Advanced Technology Center, Palo Alto, CA.

\section{$\underline{\text { References }}$}

${ }^{1}$ Koury, J.L., Kim, T.D., Tracy, J.J., and Harvey, J.A., "Continuous Fiber Composite Isogrid Structures for Space Applications," Proceedings of the 1993 Conference on Processing, Fabrication and Applications of Advanced Composites, ASM International, Materials Park, OH, 1993, pp. 193-198.

${ }^{2}$ Heard, Jr., W.L., Anderson, M.S., and Slysh, P., "An Engineering Procedure for Calculating Compressive Strength of Isogrid Cylindrical Shells with Buckled Skin," NASA TN D-8239, 1976.

${ }^{3}$ Rouse, M., and Ambur, D.R., "Damage Tolerance of a Geodesically Stiffened Structure Loaded in Axial Compression," Proceedings of the 35th AIAA/ASME/ ASCE/AHS/ASC Structures, Structural Dynamics and Materials Conference, AIAA Paper No. 94-1534, AIAA, Washington, D.C., 1994, pp. 1691-1698.

${ }^{4}$ Stein, M., "The Phenomenon of Change of Buckling Patterns in Elastic Structures," NASA Technical Report R39, 1959.

${ }^{5}$ Riks, E., Rankin, C. C., and Brogan, F. A., "On the Solution of Mode Jumping Phenomena in Thin-Walled Shell Structures," Computer Methods in Applied Mechanics and Engineering, 136(1-2), North Holland Elsevier Science, Netherlands, Sept. 1996, pp. 59-92.

${ }^{6}$ Riks, E., "Buckling Analysis of Elastic Structures: A Computational Approach," Advances in Applied Mechanics, 34, Academic Press, San Diego, CA, 1997, pp. 1-76.

${ }^{7}$ Stoll, F., and Olson, S.E., "Finite Element Investigation of the Snap Phenomenon in Buckled Plates," in Stability Analysis of Plates and Shells: A Collection of Papers in Honor of Dr. Manuel Stein, NASA-CP-1998-206280, N.F. Knight, Jr., and M.P. Nemeth, compilers, 1998, pp. 435-444.

${ }^{8}$ Waters, W. A., Jr., "Effects of Initial Geometric Imperfections on the Behavior of Graphite-Epoxy Cylinders Loaded in Compression," M.S. Thesis in Engineering Mechanics, Old Dominion University, Norfolk, VA, 1996.
${ }^{9}$ Hilburger, M.W., Starnes, J.H., Jr., and Waas, A.M., "The Response of Composite Cylindrical Shells with Cutouts and Subjected to Internal Pressure and Axial Compression Loads," Proceedings of the 39th AIAA/ASME/ASCE/AHS/ASC Structures, Structural Dynamics, and Materials Conference, AIAA-98-1768, AIAA, Washington, D.C., 1998, pp. 576-584.

${ }^{10}$ Hilburger, M.W., and Starnes, J.H., Jr., "Effects of Imperfections on the Buckling Response of Compression-Loaded Composite Shells," Proceedings of the 41st AIAA/ASME/ASCE/AHS/ASC Structures, Structural Dynamics, and Materials Conference on disk (CD-ROM), AIAA-2000-1387, AIAA, Reston, VA, 2000.

${ }^{11}$ Rose, C.A., Young, R.D., and Starnes, J.H., Jr., "The Nonlinear Response of Cracked Aluminum Shells Subjected to Combined Loads," Proceedings of the 42nd AIAA/ASME/ASCE/AHS/ASC Structures, Structural Dynamics, and Materials Conference on disk (CD-ROM), AIAA-2001-1395, AIAA, Reston, VA, 2001.

${ }^{12}$ Ziegler, H., "Principles of Structural Stability," Blaisdell Publishing, Co., Waltham, MA, 1968, pp. 9193.

${ }^{13}$ Rankin, C.C., Brogan, F.A., Loden, W.A., and Cabiness, H.D., "STAGS User Manual - Version 4.0," Lockheed Martin Missiles and Space Co., Inc., Report LMSC P032594, Palo Alto, CA, 1999.

${ }^{14}$ Rankin, C.C., and Brogan, F.A., "The Computational Structural Mechanics Testbed Structural Element Processor ES5: STAGS Shell Element," NASA Contractor Report 4358, 1991.

${ }^{15}$ Park, K.C., “An Improved Stiffly Stable Method for the Direct Integration of Nonlinear Structural Dynamics," Journal of Applied Mechanics, 42, American Society of Mechanical Engineers, New York, NY, June 1975, pp. 464-470.

${ }^{16}$ Rankin, C., and Brogan, F., Improved Plasticity and Imperfections in the STAGSC-1 Computer Code Phase 2: Implementation, LMSC-F396402, Palo Alto, CA, 1990. 
Table 1: IM7/977-2 mechanical properties

\begin{tabular}{ccccc}
\hline \hline & \multicolumn{2}{c}{ Elastic Properties } & & Density \\
$\mathrm{E}_{1}$ & $\mathrm{E}_{2}$ & $\mathrm{G}_{12}$ & $\mathrm{v}_{12}$ & $\rho$ \\
\hline $23.85 \mathrm{Msi}$ & $1.10 \mathrm{Msi}$ & $0.8 \mathrm{Msi}$ & 0.25 & $0.0582 \mathrm{lb} . / \mathrm{in} .^{3}$ \\
$164.44 \mathrm{GPa}$ & $7.58 \mathrm{GPa}$ & $5.516 \mathrm{GPa}$ & 0.25 & $1613.8 \mathrm{~kg} / \mathrm{m}^{3}$
\end{tabular}

Table 2: Eigenvalues and vibration frequencies at equilibrium state $S_{s}$ at $\Delta=1.762$

\begin{tabular}{cccc}
\hline \hline Mode & $\begin{array}{c}\text { Eigenvalue of } \boldsymbol{K}_{\text {TAN }} \\
e\end{array}$ & $\begin{array}{c}\text { Projected critical shortening } \\
\Delta\end{array}$ & $\begin{array}{c}\text { Vibration frequency } \\
f\end{array}$ \\
\hline 1 & 0.0010 & 1.764 & $76.6 \mathrm{~Hz}$ \\
2 & 0.4946 & 2.633 & $1307 \mathrm{~Hz}$ \\
\hline \hline
\end{tabular}

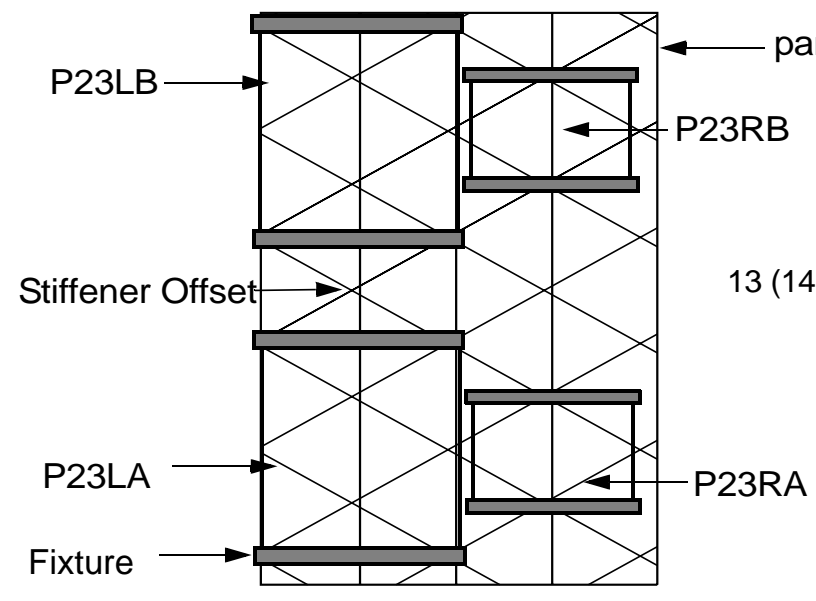

(a) Schematic of specimens cut from panel P23
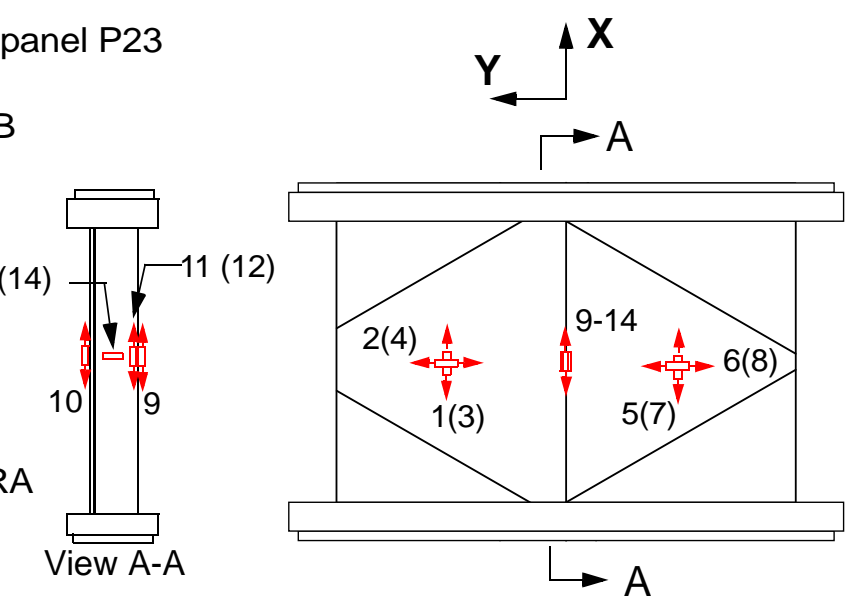

(b) Strain gage pattern P23RA \& P23RB

Fig. 1 Isogrid specimens and strain gage pattern for single axial stiffener panels 


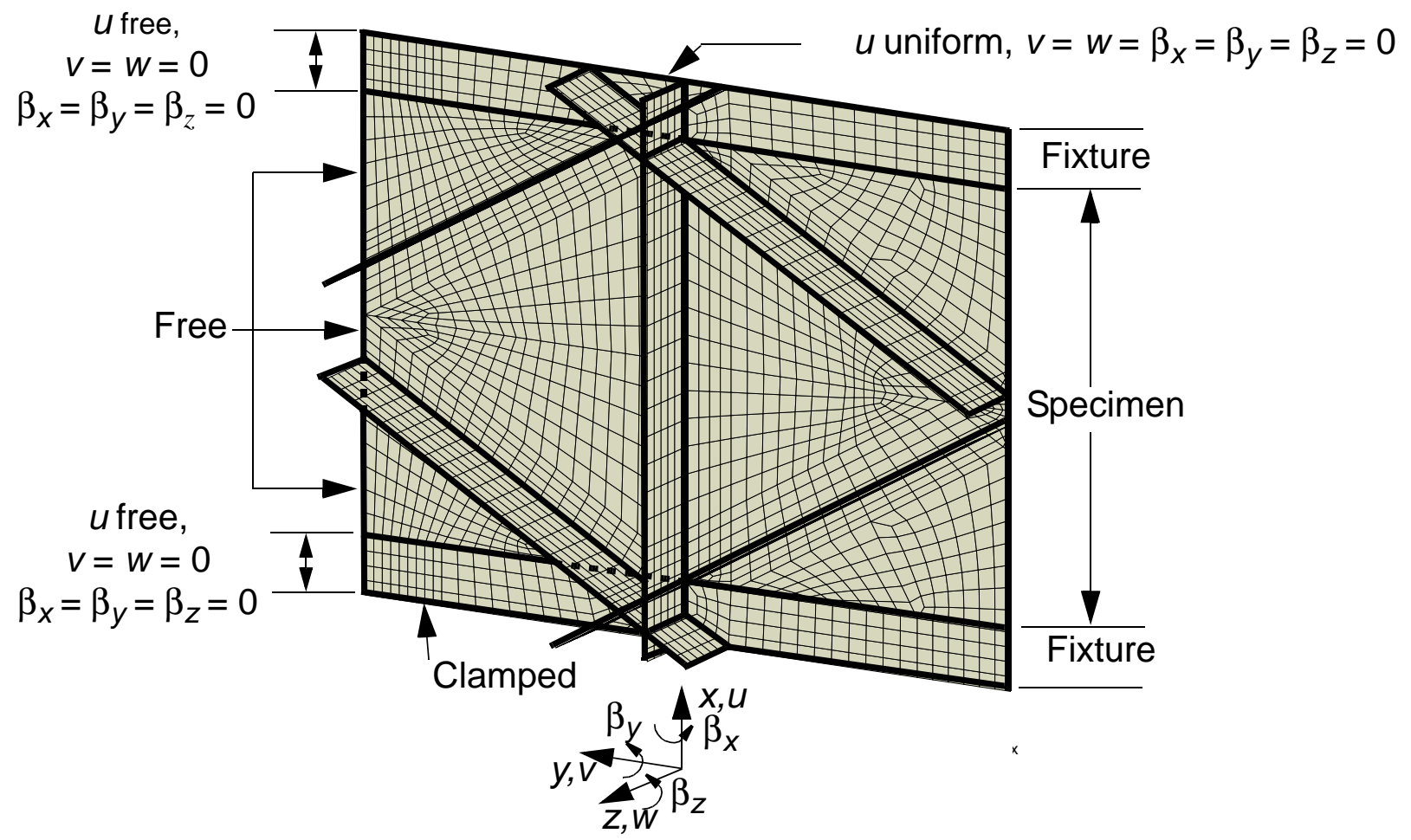

Fig. 2 Finite element model of isogrid panel P23RA

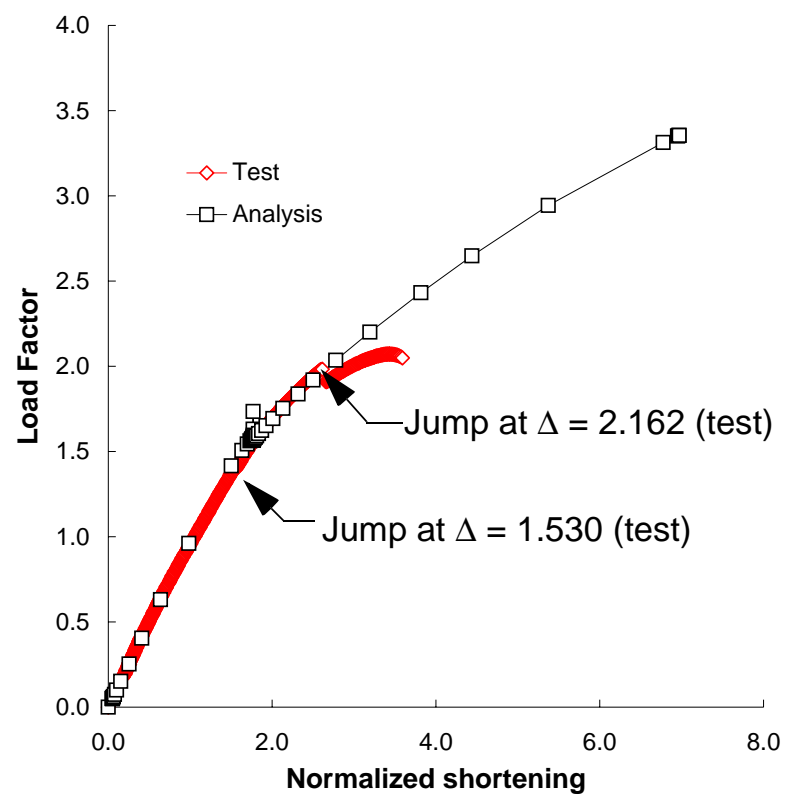

(a) Over entire analysis range

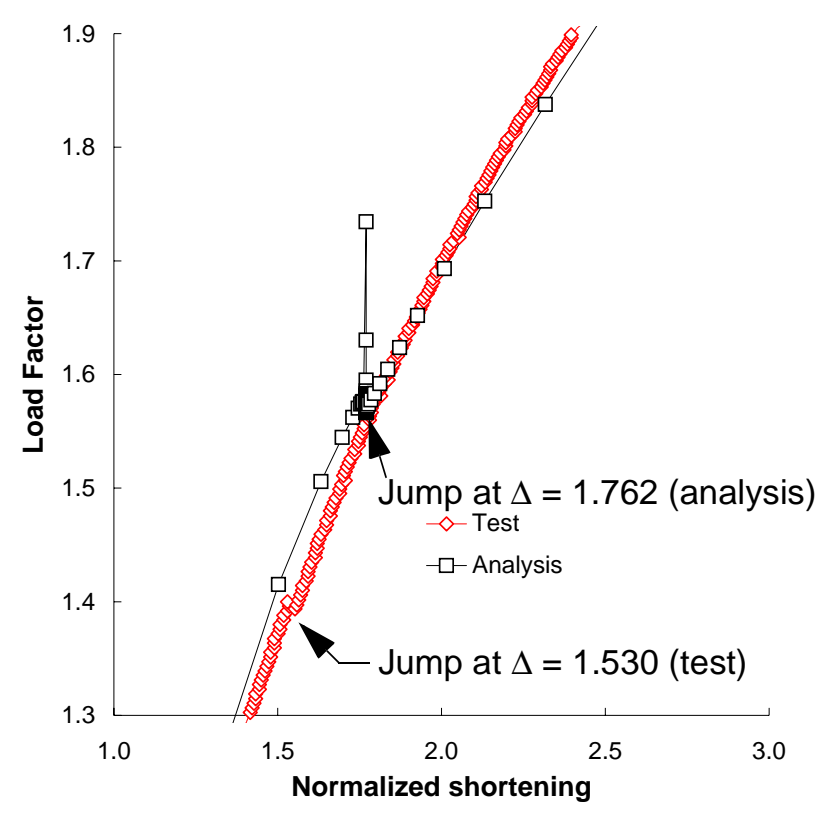

(b) Magnification near mode jump

Fig. 3 Plots of the load factor versus normalized end shortening from test and analysis 


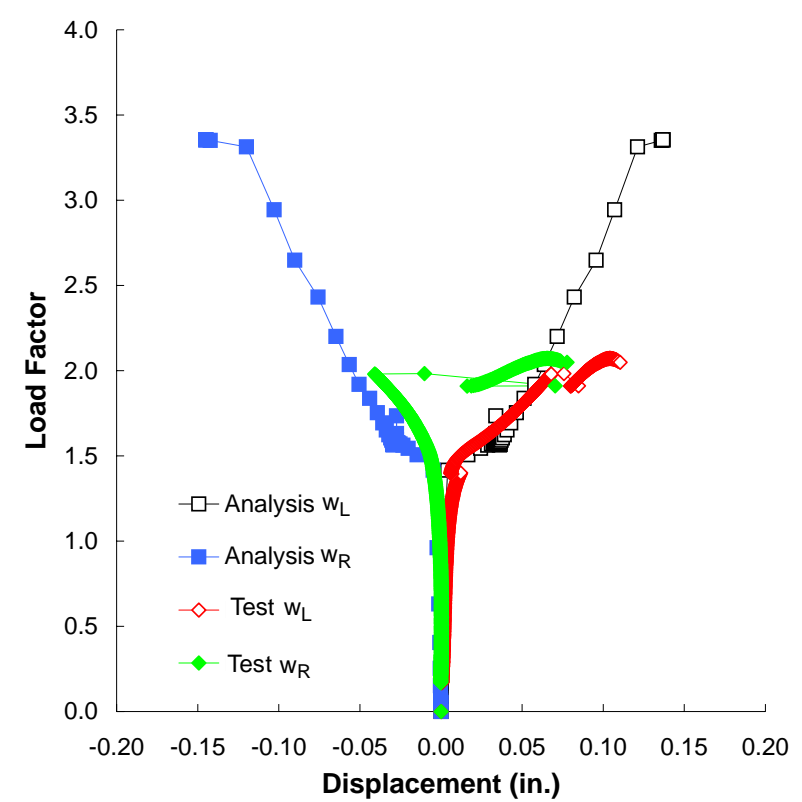

(a) Over entire load range

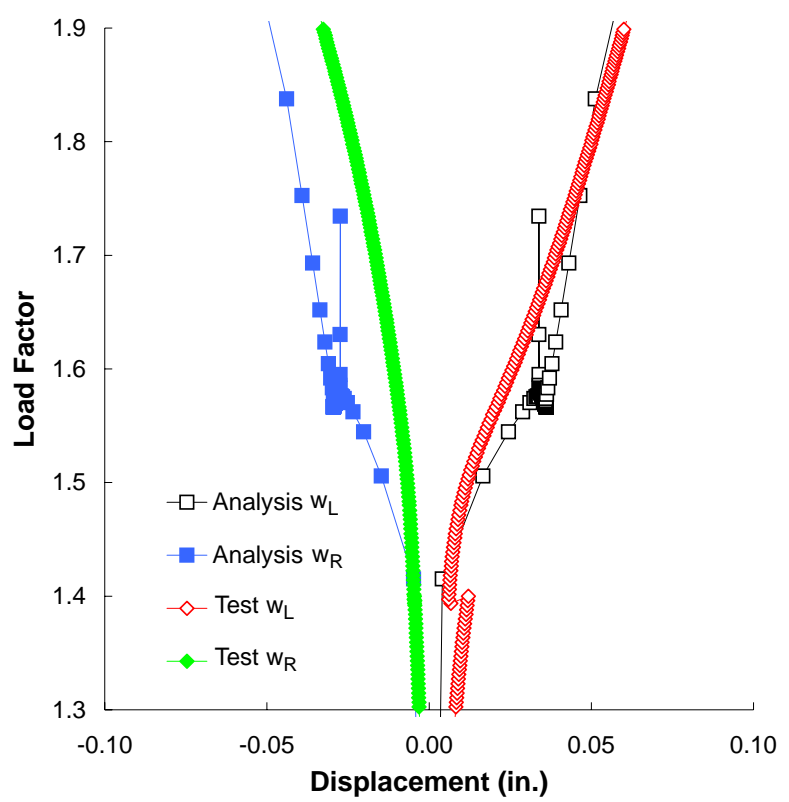

(b) Magnification near mode jump

Fig. 4 Plots of the load factor versus lateral deflection of the left side skin cell and right side skin cell

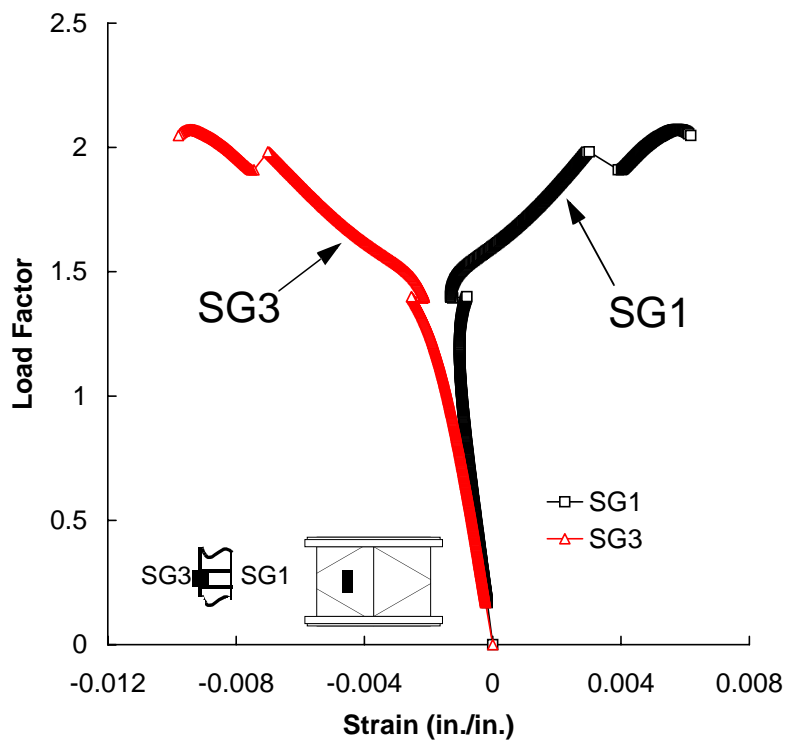

(a) Left side gages SG1 and SG3

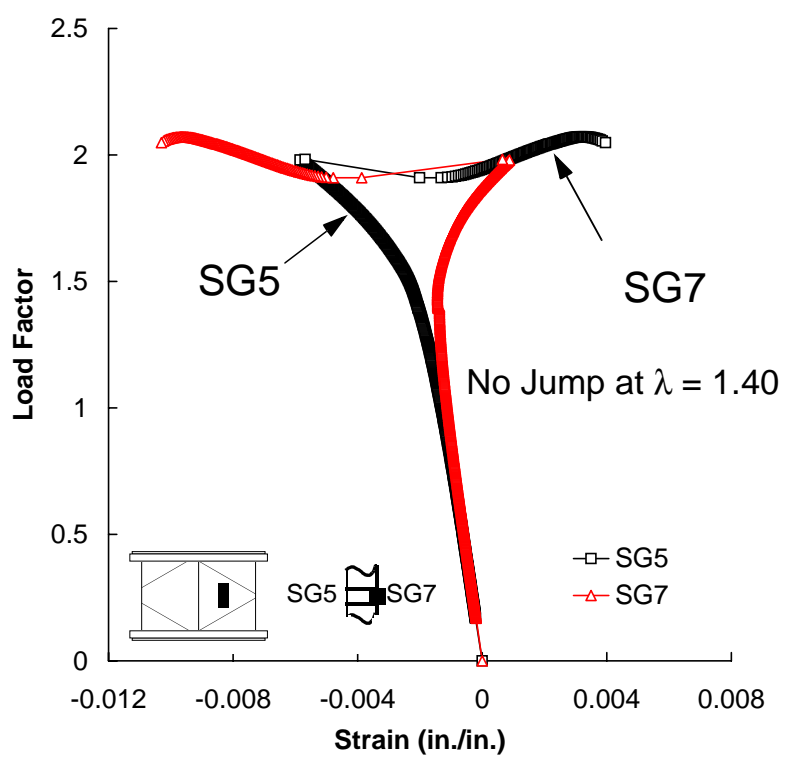

(b) Right side gages SG5 and SG7

Fig. 5 Axial strains from back-to-back gages bonded to the skin 


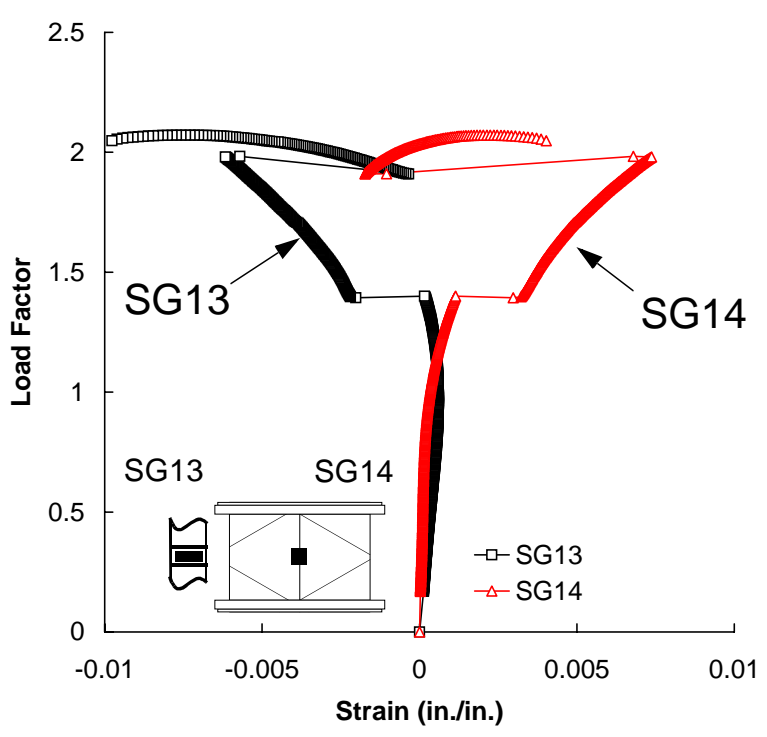

Fig. 6 Transverse strains from back-to-back strain gages SG13 and SG14

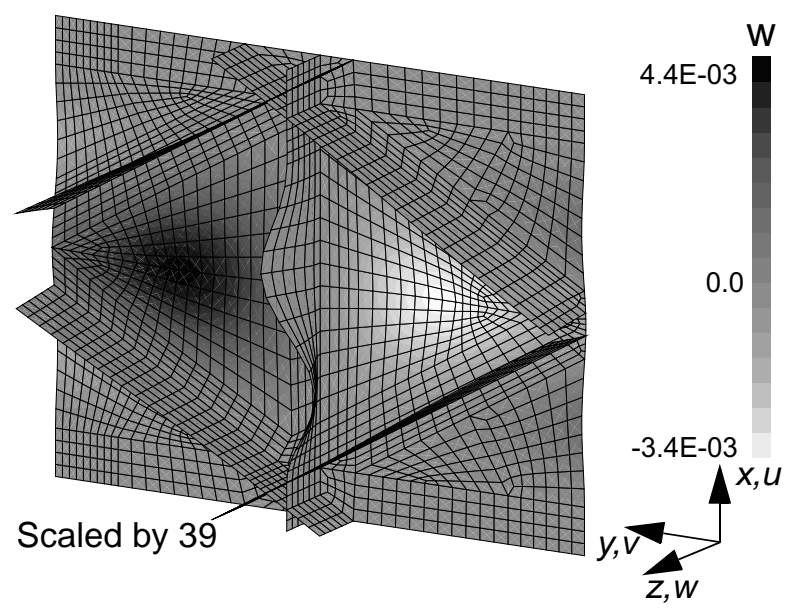

Fig. 7 Initial geometric imperfection configuration
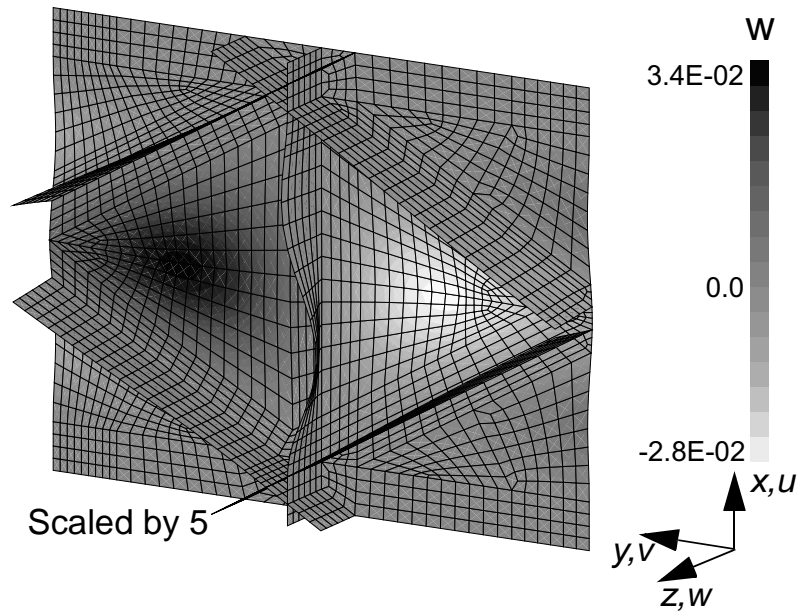

Fig. 8 Equilibrium configuration $S_{s}$ at $\Delta=1.762$

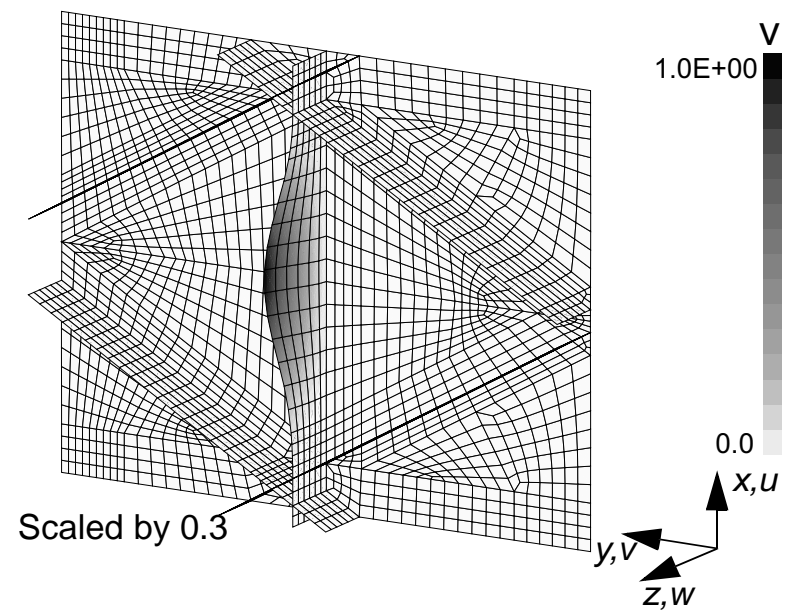

(a) Vibration mode 1 where $f=77 \mathrm{~Hz}$

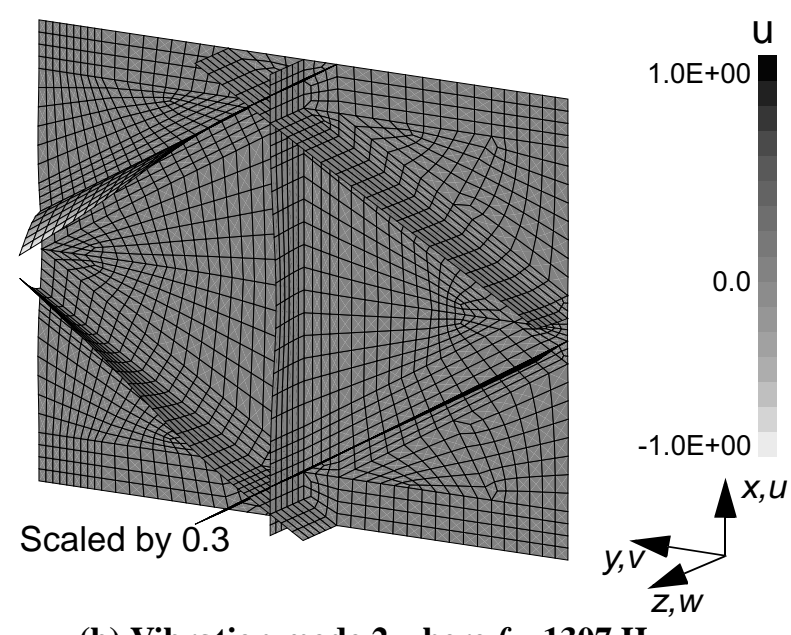

(b) Vibration mode 2 where $f=1307 \mathrm{~Hz}$

Fig. 9 Shape of vibration modes at equilibrium state $S_{s}$ at $\Delta=\mathbf{1 . 7 6 2}$
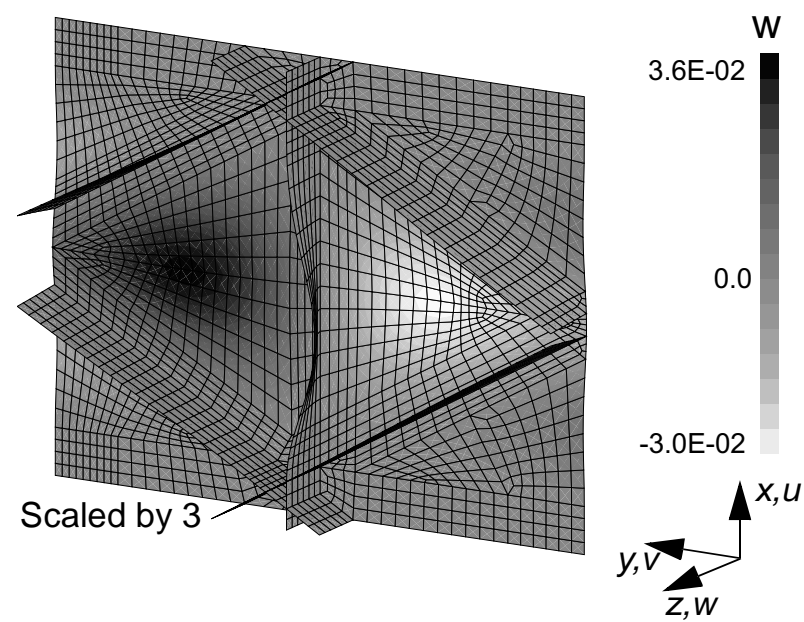

Fig. 10 Equilibrium state $S_{1}$ at $\Delta=1.7702$ following the mode jump 